\title{
三种抗坏血酸糖苷的合成及其 $\alpha$-糖苷酶抑制活性
}

\author{
马济美* 谢凌云王龙文陈杭炜曾贞 \\ 陈浩 江 洪* \\ (华中农业大学理学院化学系 武汉 430070)
}

\begin{abstract}
摘要 $2-O-\beta-D$-葡萄糖基- $L$-抗坏血酸(AA- $2 \beta \mathrm{G}$ )是从枸杞中分离得到的一种天然抗坏血酸糖苷化合物, 通过化学方法合 成了 $\mathrm{AA}-2 \beta \mathrm{G}$ 及其两种类似物, 并用商品化的阿卡波糖作为阳性对照, 研究了三种抗坏血酸糖苷对酿酒酵母 $\alpha$-葡萄糖 苷酶和猪胰腺 $\alpha$-淀粉酶的活性影响. 结果显示, 三种抗坏血酸糖苷对 $\alpha$-葡萄糖苗酶有显著的抑制作用, 对 $\alpha$-淀粉酶抑 制作用不明显，说明这三种抗坏血酸糖苷具有作为 $\alpha$-糖苷酶抑制剂开发的潜质.
\end{abstract}

关键词 $2-O-\beta-D$-葡萄糖基 $L-$-抗坏血酸; 类似物; 合成; $\alpha$-葡萄糖苷酶; $\alpha$-淀粉酶; 抑制剂

\section{Synthesis and $\alpha$-Glycosidase Inhibitory Activity of Three Ascorbic Acid Glycosides}

\author{
Ma, Jimei* Xie, Lingyun Wang, Longwen $\quad$ Chen, Hangwei \\ Zeng, Zhen Chen, Hao Jiang, Hong* \\ (Department of Chemistry, College of Science, Huazhong Agricultural University, Wuhan 430070)
}

\begin{abstract}
O-(\beta-D$-Glucopyranosyl $)-L$-ascorbic acid, a natural $\beta$-glycoside with ascorbic acid moiety present in $L y c i u m$ barbarum, and its two analogues were chemically synthesized. Their inhibitory effect on yeast $\alpha$-glucosidase and $\alpha$-amylase were investigated with commercially available acarbose as positive control. The biological assays demonstrated the distinct inhibitory effect of these compounds on $\alpha$-glucosidase activity and weak inhibitory activity against pancreatic $\alpha$-amylase. The kinetic study indicated that these compounds were competitive inhibitors. These results imply that these three $\beta$-glycosides have the potential to be developed as $\alpha$-glucosidase inhibitors.

Keywords $2-O-(\beta$ - $D$-glucopyranosyl)- $L$-ascorbic acid; analogue; synthesis; $\alpha$-glucosidase; $\alpha$-amylase; inhibitor
\end{abstract}

糖尿病是一种代谢紊乱疾病, 已成为三大严重威胁 人类健康的慢性疾病之一 ${ }^{[1]}$. 根据 2014 年国际糖尿病联 合会的统计, 全世界糖尿病患者超过 4.5 亿人, 其中 II 型糖尿病(非胰岛素依耐性糖尿病)占比超过 $90 \%{ }^{[2]}$. 在 治疗 II 型糖尿病的药物中, $\alpha$-葡萄糖苷酶抑制剂如阿卡 波糖等疗效非常显著, 但是阿卡波糖也常常会引起腹 鸣、腹胀、腹痛等副作用 ${ }^{[3]}$, 因此寻求更高效安全低副 作用的抗糖尿病药物一直是药物研究的重要目标 ${ }^{[4]}$.

枸杞果实是一种传统的中药, 在中国和一些亚洲国 家已经当作保健品食用了两千多年. 研究发现, 枸杞具 有丰富的药理活性, 如抗氧化、降血压、降血糖等效 果 ${ }^{[5]}$. 2- $O-\beta-D$ - 葡萄糖基- $L$-抗坏血酸(AA- $2 \beta \mathrm{G}$ )是一种天
然的抗坏血酸衍生物, 最早由日本学者 Toyada-ono 从宁 夏枸杞中分离得到 ${ }^{[6]}, \mathrm{AA}-2 \beta \mathrm{G}$ 在体内只能被小肠中的 $\beta$ 糖苷酶水解，具有缓释抗坏血酸的作用，能达到持续清 除自由基和抗氧化的效果 ${ }^{[7]}$, 还能抑制 Hela 细胞增殖生 $k^{[8]}$. 根据 $\mathrm{AA}-2 \beta \mathrm{G}$ 分子的结构特点, 我们推测 $\mathrm{AA}-2 \beta \mathrm{G}$ 有可能会与食物中糖类化合物在结合 $\alpha$-糖苷酶时产生 竞争，起到抑制血糖急剧升高的作用. 为了证实这个推 测, 我们合成了 $\mathrm{AA}-2 \beta \mathrm{G}$ 及其两种类似物 $2-O-\beta-D$-半乳 糖基- $L$-抗坏血酸(AA-2 $\beta \mathrm{Gal})$ 和 3- $O-\beta-D$-葡萄糖基- $L$-抗 坏血酸(AA-3 $3 \mathrm{G}$ ), 并研究了它们在体外对 $\alpha$-糖苷酶的 影响.

\footnotetext{
* Corresponding authors. E-mail: majimei@mail.hzau.edu.cn; jianghong@mail.hzau.edu.cn Received December 11, 2016; revised January 20, 2017; published online February 20, 2017. 2014QC007).

国家自然科学基金(No. 21402056)和中央高校基本科研业务费专项资金(No. 2014QC007)资助项目.
}

Project supported by the National Natural Science Foundation of China (No. 21402056) and the Fundamental Research Funds for the Central Universities (No. 


\section{1 结果与讨论}

AA-2 $\beta G$ 的合成路线如 Scheme 1 所示. 由于抗坏血 酸的 2 位羟基容易与羰基形成分子内氢键, 导致 2 位羟 基反应活性比其他羟基低, 因此制备 $\mathrm{AA}-2 \beta \mathrm{G}$ 时需先保 护抗坏血酸的 3、5、6 位羟基 ${ }^{[9]}$. 以商品化的 5,6- $O$-异 丙叉基- $L$-抗坏血酸 1 为原料, 先后尝试了使用叔丁基二 甲基硅基(TBS)、芐氧羰基 $(\mathrm{Cbz})$ 和苄基(Bn)保护 3 位羟 基. 以 $\mathrm{Cbz}$ 为保护基时, 在碳酸氢钠 $/ \mathrm{THF} / \mathrm{H}_{2} \mathrm{O}$ 、碳酸钾 / $\mathrm{THF} / \mathrm{H}_{2} \mathrm{O}$ 、碳酸钾/DMSO/THF 等条件下, 3- $O$-苠氧羰 基-5,6- $O$-异丙叉基- $L$-抗坏血酸产率均较低; 控制试剂 的用量, 在碳酸钾和 $N, N$-二甲基甲酰胺(DMF)中, TBS 能选择性地保护化合物 1 的 3 位羟基, 但遗憾的是在随 后以溴代糖为糖基供体, 碳酸钾反应条件中不稳定, TBS 基团会发生脱除导致糖苷化反应发生在抗坏血酸 的 3 位羟基(经核磁氢谱确认); 最后我们使用酸碱耐受 性较高的茮基保护基，控制保护基试剂的用量和反应时 间，以较高的产率(81\%)得到化合物 2 .
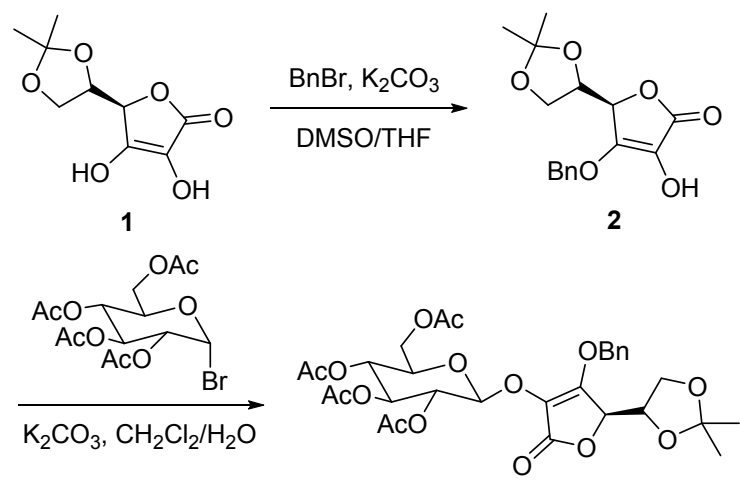

3

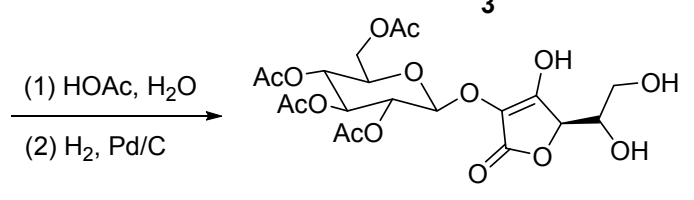

4

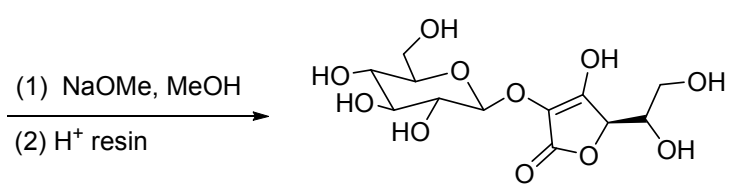

AA-2 $\beta G(5)$

图式 1 AA-2 $2 \beta$ G 的合成路线

Scheme 1 Synthetic route to AA-2 $\beta$ G

在糖苷化反应中, 我们首先尝试了 Schmidt 给体法, 即以三氯乙酰亚胺酯为糖基供体, 但此类反应在 Lewis 酸催化条件(TMSOTf 或三氟化嗍乙醚)下进行, 得到的 反应产物复杂, 难以纯化. 后改用溴代糖作为糖基供体, 在碱性条件下发生糖苷化, 以碳酸钾为碱、DMF 为溶剂
的常规条件下，但反应产率依然非常低，分析原因可能 是碳酸钾在 DMF 中溶解度较低, 而糖基受体 2 位羟基 本身活性偏低所致. 最后通过探索条件, 发现使用二氯 甲烷和水作为溶剂, 同时在相转移催化剂四丁基溴化铵 作用下，反应效率大为提高，以 $80 \%$ 的产率得到了化合 物 3.

化合物 3 水解条件也必须注意, 为避免糖苷键在强 酸性条件下断裂，选择用醋酸水溶液加热条件下脱除异 丙叉保护基，然后氢化脱除茮基得到化合物 4, 最后用 甲醇钠脱除乙酰基得到 AA-2 $\beta G$ (Scheme 1). 与文献合 成路线方法 ${ }^{[6]}$ 相比 $(13 \%)$, 本路线大大提高了反应总收 率 $(53 \%)$. 以 1 -溴-2,3,4,6-四乙酰基半乳糖为原料, 用相 同的方法可以制备 AA-2 $\beta$ Gal(总产率 51\%, Scheme 2); AA-3 $\beta$ G 则通过 5,6- $O$-异丙叉基- $L$-抗坏血酸直接与 1 -溴 -2,3,4,6-四乙酰基葡萄糖反应后，依次脱除保护基得到 (总产率 51\%, Scheme 3).<smiles>CC1(C)OC[C@H](C2OC(=O)C(O)=C2Br)O1</smiles>

2

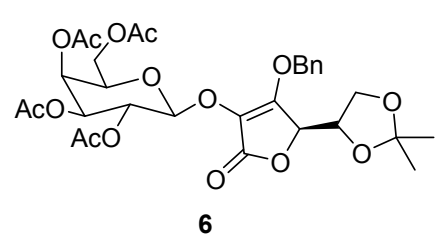

(1) $\mathrm{HOAc}, \mathrm{H}_{2} \mathrm{O}$

(2) $\mathrm{H}_{2}, \mathrm{Pd} / \mathrm{C}$

(3) $\mathrm{NaOMe}, \mathrm{MeOH}$

(4) $\mathrm{H}^{+}$resin<smiles></smiles>

图式 $2 \mathrm{AA}-2 \beta \mathrm{Gal}$ 的合成路线

Scheme 2 Synthetic route to AA-2 $\beta G a l$

所得化合物经过 NMR 和 HRMS 确认结构, 然后分 别进行了对酿酒酵母 $\alpha$-葡萄糖苷酶和 $\alpha$-猪胰腺淀粉酶 活性的测试. 结果显示, 三种化合物均对 $\alpha$-葡萄糖苷酶 有明显的抑制活性，抑制率与浓度成正相关性，通过计 算得出 $\mathrm{AA}-2 \beta \mathrm{G} 、 \mathrm{AA}-2 \beta \mathrm{Gal}$ 和 $\mathrm{AA}-3 \beta \mathrm{G}$ 的 $\mathrm{IC}_{50}$ 值分别为 $0.98,1.15,63.67 \mathrm{mmol} / \mathrm{L}$ (表 1). 其中, $\mathrm{AA}-2 \beta \mathrm{G}$ 和 $\mathrm{AA}-2 \beta \mathrm{Gal}$ 的 $\mathrm{IC}_{50}$ 值小于阿卡波糖阳性对照组(4.54 $\mathrm{mmol} / \mathrm{L})$, 说明 AA- $2 \beta \mathrm{G}$ 和 AA- $2 \beta \mathrm{Gal}$ 对酿酒酵母 $\alpha$-葡萄 糖苷酶的抑制能力高于阿卡波糖.

我们通过动力学研究了三种抗坏血酸糖苷化合物 对 $\alpha$-葡萄糖苷酶的抑制类型. 根据不同浓度下的三种化 


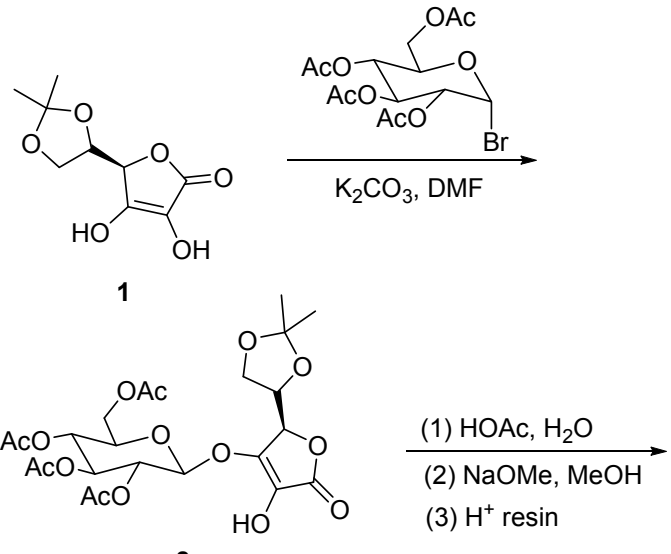

8<smiles>O=C1OC(C(O)CO)C(OC2OC(CO)C(O)C(O)C(O)C2O)=C1O</smiles>

AA-3ßG (9)

图式 $3 \mathrm{AA}-3 \beta \mathrm{G}$ 的合成路线

Scheme 3 Synthetic route to AA-3 $\beta$ G

表 1 测试化合物对 $\alpha$-葡萄糖苷酶的半抑制浓度

Table $1 \mathrm{IC}_{50}$ value of the tested compounds against $\alpha$-glucosidase

\begin{tabular}{cc}
\hline 化合物 & $\mathrm{IC}_{50} /\left(\mathrm{mmol} \cdot \mathrm{L}^{-1}\right)$ \\
\hline 阿卡波糖 & $4.54 \pm 0.23$ \\
$\mathrm{AA}-2 \beta \mathrm{G}$ & $0.98 \pm 0.12$ \\
$\mathrm{AA}-2 \beta \mathrm{Gal}$ & $1.15 \pm 0.21$ \\
$\mathrm{AA}-3 \beta \mathrm{G}$ & $63.67 \pm 2.60$ \\
\hline
\end{tabular}

合物水解 $p$ NPG 的速率，作出 Linewaever-Burk 图如图 1 所示, 随着浓度的增加, 米氏常数 $K_{\mathrm{m}}$ 变大, 最大速率保 持不变, 说明三种化合物为竞争性抑制, 通过 Dixon 方 程计算得到 AA-2 $\beta \mathrm{G} 、 \mathrm{AA}-2 \beta \mathrm{Gal}$ 和 AA-3 $3 \mathrm{G}$ 的抑制常数 $K_{\mathrm{i}}$ 分别为 $1.98,7.25$ 和 $19.56 \mathrm{mmol} / \mathrm{L}$.

$\mathrm{AA}-2 \beta \mathrm{G} 、 \mathrm{AA}-2 \beta \mathrm{Gal}$ 和 $\mathrm{AA}-3 \beta \mathrm{G}$ 对猪胰腺 $\alpha$-淀粉酶 的抑制活性结果如图 2 所示. 作为阳性对照的阿卡波糖, 在浓度为 $0.015 \mathrm{mmol} / \mathrm{L}$ 时, 对 $\alpha$-淀粉酶的抑制率为 $42.7 \%$, 而当浓度增加到 $0.077 \mathrm{mmol} / \mathrm{L}$ 时, 抑制率达到 了 $90.6 \%$. 而 AA- $2 \beta \mathrm{G} 、 \mathrm{AA}-2 \beta \mathrm{Gal}$ 和 AA- $3 \beta \mathrm{G}$ 对 $\alpha$-淀粉 酶的影响则较小, 在浓度为 $0.015 \mathrm{mmol} / \mathrm{L}$ 时抑制率分别 为 $5.9 \% 、 19.4 \%$ 和 $4.6 \%$, 浓度增加到 $0.77 \mathrm{mmol} / \mathrm{L}$ 时也 仅为 $12.9 \% 、 25.3 \%$ 和 $5.9 \%$ 。

阿卡波糖作为 $\alpha$-糖苷酶抑制剂, 是一种有效的糖尿 病治疗药物, 然而, 服用阿卡波糖常常伴有胃肠胀气和 肠鸣音及腹痛、腹胀、腹泻等副作用, 这可能是由于阿 卡波糖对 $\alpha$-淀粉酶的强抑制作用, 导致未消化的淀粉在 肠道内聚集, 被肠道菌群发酵释放出气体和低分子量物 质引起的 ${ }^{[10]}$. 相比之下, $A A-2 \beta G 、 A A-2 \beta G a l$ 和 $A A-3 \beta G$
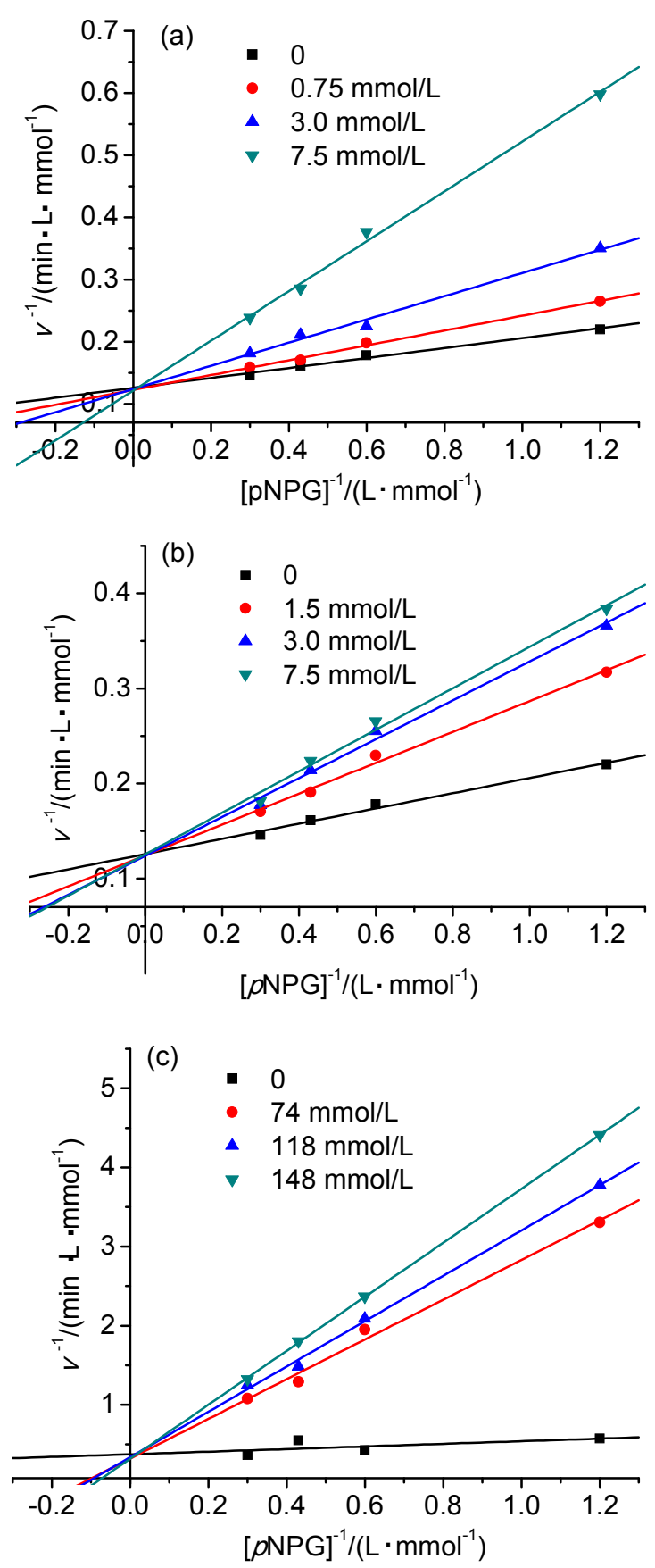

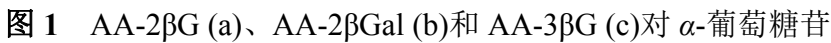
酶的抑制类型曲线

Figure 1 Determination of the inhibitory type of AA-2 $\beta \mathrm{G}$ (a), AA-2 $\beta$ Gal (b) and AA-3 $\beta$ G (c) on $\alpha$-glucosidase

对猪胰腺 $\alpha$-淀粉酶的抑制效果远不如阿卡波糖，如果作 为 $\alpha$-糖苷酶抑制剂药物开发, 这三种糖苷可能不会引起 以上不良反应.

\section{2 结论}

通过化学方法合成了 $\mathrm{AA}-2 \beta \mathrm{G} 、 \mathrm{AA}-2 \beta \mathrm{Gal}$ 和 AA$3 \beta \mathrm{G}$ ，并发现三种化合物对 $\alpha$-葡萄糖苷酶具备明显的抑 


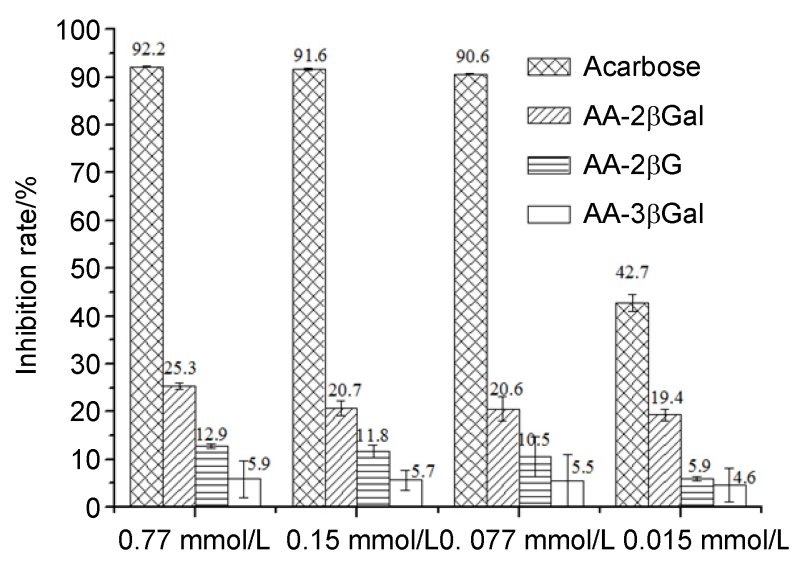

图 2 测试物对 $\alpha$-淀粉酶的抑制活性

Figure 2 Inhibitory activity of the tested compounds on $\alpha$-amylase

制能力, 抑制类型为竞争性抑制, 而对 $\alpha$-淀粉酶影响很 小, 说明这类抗坏血酸糖苷化合物可以作为 $\alpha$-葡萄糖苷 酶抑制剂. 尤其是 AA- $2 \beta \mathrm{G}$, 本身是一种天然安全的保 健品成分, 具有作为低副作用抗糖尿药物开发的潜质, 同时本研究内容也为开发传统保健品枸杞降血糖功能 提供了新的线索.

\section{3 实验部分}

\section{1 仪器与试剂}

核磁共振仪(AV400, AV600, USA, TMS 为内标); 质 谱仪 (Agilent 6100 ESI MS, USA); 高分辨质谱仪 (Agilent 6540 ESI MS, USA; Bruker MicroTof Q II, USA); 葡萄糖苷酶(EC 3.2.1.20, Sigma), 淀粉酶(EC 3.2.1.1, Sigma), 其余试剂为市售化学纯或分析纯产品.

\section{2 化合物的合成}

\subsubsection{3- $O$ - 芐基-5,6-异丙叉基- $L$-抗坏血酸(2)的合成}

5,6- $O$-异丙叉基- $L$-抗坏血酸 1 (1.5 g, $6.93 \mathrm{mmol}$ )溶 于四氢呋喃/二甲亚砜 $(24 \mathrm{~mL}, V / V=1: 1)$ 中, 加入碳酸 钾 $(0.957 \mathrm{~g}, 6.93 \mathrm{mmol})$, 然后逐滴滴加茮溴 $(4.05 \mathrm{~mL}$, $34.65 \mathrm{mmol})$, 滴加完毕, 室温 $\left(25^{\circ} \mathrm{C}\right)$ 条件下反应 $0.5 \mathrm{~h}$, 反应进程薄层色谱(TLC)检测跟踪. 反应结束后减压蒸 除溶剂四氢呋喃, 加入二氯甲烷 $(20 \mathrm{~mL})$, 用柠檬酸水溶 液洗涤数次, 至体系呈中性, 有机相用 $\mathrm{MgSO}_{4}$ 干燥, 过 滤, 滤液浓缩, 硅胶柱层析 $[V($ 乙酸乙酯 $): V($ 石油醚 $)=$ 1：4]分离得到 $1.72 \mathrm{~g}$ 白色固体 $\mathbf{2}^{[9 \mathrm{a}]}$, 产率 $81 \%$. m.p. $200 \sim 201{ }^{\circ} \mathrm{C}$ (文献值 ${ }^{[11]} 202.7 \sim 203.5{ }^{\circ} \mathrm{C}$ ); ${ }^{1} \mathrm{H}$ NMR $\left(\mathrm{CDCl}_{3}, 600 \mathrm{MHz}\right) \delta: 7.42 \sim 7.34(\mathrm{~m}, 5 \mathrm{H}), 5.53(\mathrm{~d}, J=11.8$ $\mathrm{Hz}, 1 \mathrm{H}), 5.49$ (d, $J=11.8 \mathrm{~Hz}, 1 \mathrm{H}), 4.57$ (d, $J=3.7 \mathrm{~Hz}$, $1 \mathrm{H}), 4.26(\mathrm{dt}, J=6.7,3.8 \mathrm{~Hz}, 1 \mathrm{H}), 4.10(\mathrm{dd}, J=8.5,6.9$ $\mathrm{Hz}, 1 \mathrm{H}), 4.02$ (dd, $J=8.5,6.8 \mathrm{~Hz}, 1 \mathrm{H}), 1.38$ (s, 3H), 1.35 (s, 3H).
3.2.2 2- $O$ - $(2,3,4,6$-四乙酰基- $\beta-D$ - 葡匋糖基)-3- $O$ - 芐 基-5,6- $O$-异丙叉基-L-抗坏血酸(3)的合成

将化合物 $2(1.72 \mathrm{~g}, 5.6 \mathrm{mmol})$ 加入到二氯甲烷/水 (34 mL, $V: V=1: 1)$ 的混合溶液中, 室温下搅拌均匀后 加入溴代五乙酰葡萄糖 $(2.77 \mathrm{~g}, 6.72 \mathrm{mmol})$, 随之加入碳 酸钾 $(0.93 \mathrm{~g}, 6.72 \mathrm{mmol})$ 和四丁基溴化铵 $(2.16 \mathrm{~g}, 6.72$ $\mathrm{mmol}), 25{ }^{\circ} \mathrm{C}$ 下搅拌反应 $5 \mathrm{~h}$. 反应停止, 将水相与有机 相分离, 有机相用柠檬酸水溶液洗涤 2 次, 用 $\mathrm{MgSO}_{4}$ 干 燥, 过滤, 滤液浓缩, 残余物经硅胶柱层析 $[V($ 乙酸乙 酯)： $V$ (石油醚 $)=1: 3$ ]分离得到 $2.86 \mathrm{~g}$ 白色固体 3 , 产 率 $80 \%$. m.p. $138 \sim 140{ }^{\circ} \mathrm{C}$; $[\alpha]_{\mathrm{D}}^{20}-4.73$ (c 1.0, $\mathrm{CHCl}_{3}$ ); ${ }^{1} \mathrm{H}$ NMR $\left(\mathrm{CDCl}_{3}, 400 \mathrm{MHz}\right) \delta: 7.41 \sim 7.34(\mathrm{~m}, 5 \mathrm{H}), 5.55$ $(\mathrm{d}, J=11.6 \mathrm{~Hz}, 1 \mathrm{H}), 5.41$ (d, $J=11.6 \mathrm{~Hz}, 1 \mathrm{H}), 5.39$ (d, $J=$ $8.0 \mathrm{~Hz}, 1 \mathrm{H}), 5.28(\mathrm{t}, J=9.5 \mathrm{~Hz}, 1 \mathrm{H}), 5.17 \sim 5.08(\mathrm{~m}, 2 \mathrm{H})$, $4.56(\mathrm{~d}, J=3.3 \mathrm{~Hz}, 1 \mathrm{H}), 4.30 \sim 4.24(\mathrm{~m}, 2 \mathrm{H}), 4.14(\mathrm{dd}, J=$ 12.4, $2.3 \mathrm{~Hz}), 4.08$ (dd, $J=8.5,6.8 \mathrm{~Hz}, 1 \mathrm{H}), 3.99$ (dd, $J=$ $8.5,6.8 \mathrm{~Hz}, 1 \mathrm{H}), 3.76$ (ddd, $J=10.0,4.4,2.3 \mathrm{~Hz}, 1 \mathrm{H}$ ), 2.11 (s, 3H), 2.02 (s, 3H), 2.01 (s, 3H), 1.97 (s, 3H), 1.37 (s, $3 \mathrm{H}), 1.34(\mathrm{~s}, 3 \mathrm{H}) ;{ }^{13} \mathrm{C} \mathrm{NMR}\left(\mathrm{CDCl}_{3}, 100 \mathrm{MHz}\right) \delta: 170.4$, 169.9 (2C), 169.5, 167.8, 158.5, 135.2, 128.9, 128.7, 128.2, 118.1, 110.4, 98.3, 74.9, 74.5, 73.9, 72.4, 72.1, 71.0, 68.1, 65.2, 61.4, 25.8, 25.6, 20.8, 20.6 (2C); HRMS (ESI) calcd for $\mathrm{C}_{30} \mathrm{H}_{36} \mathrm{O}_{15} \mathrm{Na}[\mathrm{M}+\mathrm{Na}]^{+}$659.1946, found 659.1959.

3.2.32-O-(2,3,4,6-四乙酰基- $\beta-D$ - 葡萄糖基)- $L$-抗坏 血酸(4)的合成

将化合物 3 (2.00 g, $3.14 \mathrm{mmol})$ 溶于 $50 \mathrm{~mL}$ 体积分数 为 $80 \%$ 的 $\mathrm{CH}_{3} \mathrm{COOH}$ 水溶液, $80{ }^{\circ} \mathrm{C}$ 下加热反应 $1 \mathrm{~h}$. 冷 却, 体系中加入 $20 \mathrm{~mL}$ 乙酸乙酯, 用饱和 $\mathrm{NaHCO}_{3}$ 溶液 洗涤分液, 中和多余的 $\mathrm{CH}_{3} \mathrm{COOH}$, 有机相用 $\mathrm{MgSO}_{4}$ 干 燥, 过滤, 旋干, 柱层析 $[V$ (乙酸乙酯 $): V($ 石油醚 $)=2$ : 1]分离得到 $1.80 \mathrm{~g}$ 白色发泡状固体化合物，向该化合物 $(1.80 \mathrm{~g}, 2.83 \mathrm{mmol})$ 中加入其质量 0.02 倍质量分数为 $10 \%$ 的 $\mathrm{Pd} / \mathrm{C}$ 湿剂. 将体系抽成真空状态, 加入甲醇, 通 入氢气，室温下反应 $2.5 \mathrm{~h}$, 抽滤, 滤液旋干, 柱层析(乙 酸乙酯)得到 $1.36 \mathrm{~g}$ 白色固体 4, 产率 85\%. m.p. 163 $165{ }^{\circ} \mathrm{C} ;[\alpha]_{\mathrm{D}}^{20}-3.4\left(c 1.0, \mathrm{CH}_{3} \mathrm{OH}\right) ;{ }^{1} \mathrm{H}$ NMR (DMSO- $d_{6}$, $400 \mathrm{MHz}) \delta: 5.38 \sim 5.29(\mathrm{~m}, 2 \mathrm{H}), 4.96 \sim 4.90(\mathrm{~m}, 2 \mathrm{H})$, $4.74(\mathrm{~s}, 1 \mathrm{H}), 4.23(\mathrm{dd}, J=12.3,4.8 \mathrm{~Hz}, 1 \mathrm{H}), 4.05 \sim 4.00$ $(\mathrm{m}, 1 \mathrm{H}), 3.95(\mathrm{dd}, J=12.3,2.1 \mathrm{~Hz}, 1 \mathrm{H}), 3.78 \sim 3.74(\mathrm{~m}$, $1 \mathrm{H}), 3.46 \sim 3.41(\mathrm{~m}, 2 \mathrm{H}), 2.02(\mathrm{~s}, 3 \mathrm{H}), 2.00(\mathrm{~s}, 3 \mathrm{H}), 1.98$ $(\mathrm{s}, 3 \mathrm{H}), 1.95(\mathrm{~s}, 3 \mathrm{H}) ;{ }^{13} \mathrm{C}$ NMR (DMSO-d, $\left.100 \mathrm{MHz}\right) \delta$ : 170.1, 169.6, 169.4, 169.3, 168.6, 162.0, 117.1, 98.6, 74.8, 71.9, 71.0, 70.8, 68.6, 68.0, 61.8, 61.5, 20.5 (2C), 20.4, 20.3; HRMS (ESI) calcd for $\mathrm{C}_{20} \mathrm{H}_{26} \mathrm{O}_{15} \mathrm{Na}[\mathrm{M}+\mathrm{Na}]^{+}$ 
529.1164, found 529.1163.

\subsubsection{2- $O-\beta-D$-葡萄糖基- $L$-抗坏血酸(5)的合成}

将化合物 4 (0.51 g, $1 \mathrm{mmol})$ 溶于 $30 \mathrm{~mL}$ 甲醇中, 加 入 $\mathrm{CH}_{3} \mathrm{ONa}(21.6 \mathrm{mg}, 0.4 \mathrm{mmol})$, 室温下反应 $3 \mathrm{~h}$. 反应 结束加入 $5 \mathrm{~g}$ 的 732 阳离子交换树脂, 室温搅拌 $30 \mathrm{~min}$, 反应体系成弱酸性. 抽滤收集溶液, 旋干得到 $0.33 \mathrm{~g}$ 白 色发泡状固体 5, 产率 98\%. m.p. 62 63 ${ }^{\circ} \mathrm{C} ;[\alpha]_{\mathrm{D}}^{20}+$ 29.30 (c 1.0, $\left.\mathrm{H}_{2} \mathrm{O}\right) ;{ }^{1} \mathrm{H}$ NMR $\left(\mathrm{D}_{2} \mathrm{O}, 400 \mathrm{MHz}\right) \delta: 4.72$ (d, $J=7.8 \mathrm{~Hz}, 1 \mathrm{H}), 4.56(\mathrm{~d}, J=1.8 \mathrm{~Hz}, 1 \mathrm{H}), 4.04 \sim 4.00(\mathrm{~m}$, $1 \mathrm{H}), 3.88 \sim 3.84(\mathrm{~m}, 1 \mathrm{H}), 3.80 \sim 3.70(\mathrm{~m}, 3 \mathrm{H}), 3.55 \sim 3.40$ $(\mathrm{m}, 4 \mathrm{H}) ;{ }^{13} \mathrm{C}$ NMR $\left(\mathrm{D}_{2} \mathrm{O}, 100 \mathrm{MHz}\right) \delta: 175.4,173.9,115.8$, 102.9, 77.8, 76.2, 75.4, 72.8, 69.3, 69.3, 62.2, 60.4; HRMS (ESI) calcd for $\mathrm{C}_{12} \mathrm{H}_{19} \mathrm{O}_{11}[\mathrm{M}+\mathrm{H}]^{+} 339.0922$, found 339.0917 .

3.2.5 2- $O$-(2,3,4,6-四乙酰基- $\beta$ - $D$-半乳糖基)-3- $O$ - 芐 基-5,6- $O$-异丙叉基- $L$-抗坏血酸(6)的合成

将化合物 2 (2.45 g, $8 \mathrm{mmol})$ 加入到二氯甲烷/水(50 $\mathrm{mL}, V: V=1: 1)$ 的混合溶液中, 室温下搅拌均匀后加 入溴代四乙酰半乳糖 $(3.95 \mathrm{~g}, 9.6 \mathrm{mmol})$, 随之加入 $\mathrm{K}_{2} \mathrm{CO}_{3}(1.33 \mathrm{~g}, 9.6 \mathrm{mmol}$ )和四丁基溴化铵 $(3.1 \mathrm{~g}, 9.6$ $\mathrm{mmol}$ ), 室温 $25{ }^{\circ} \mathrm{C}$ 下搅拌反应 $5 \mathrm{~h}$. 反应停止, 将水相 与有机相分离, 有机相用柠檬酸水溶液洗涤数次, $\mathrm{MgSO}_{4}$ 干燥, 过滤, 滤液旋干, 柱层析 $[V$ (乙酸乙酯) : $V($ 石油醚 $)=1: 3$ 分离得到 $3.87 \mathrm{~g}$ 白色发泡状固体 6, 产 率 76\%. m.p. 96 98 ${ }^{\circ} \mathrm{C}$; $[\alpha]_{\mathrm{D}}^{20}+26.73\left(c 1.0, \mathrm{CHCl}_{3}\right) ;{ }^{1} \mathrm{H}$ NMR $\left(\mathrm{CDCl}_{3}, 400 \mathrm{MHz}\right) \delta: 7.40 \sim 7.3(\mathrm{~m}, 5 \mathrm{H}), 5.58(\mathrm{~d}$, $J=11.7 \mathrm{~Hz}, 1 \mathrm{H}), 5.47 \sim 5.43(\mathrm{~m}, 2 \mathrm{H}), 5.37 \sim 5.30(\mathrm{~m}, 2 \mathrm{H})$, $5.09(\mathrm{dd}, J=9.8,3.5 \mathrm{~Hz}, 1 \mathrm{H}), 4.56(\mathrm{~d}, J=3.3 \mathrm{~Hz}, 1 \mathrm{H})$, $4.27(\mathrm{td}, J=6.6,3.3 \mathrm{~Hz}, 1 \mathrm{H}), 4.19(\mathrm{dd}, J=11.2,6.6 \mathrm{~Hz}$, $1 \mathrm{H}), 4.13 \sim 4.07(\mathrm{~m}, 2 \mathrm{H}), 4.02 \sim 3.96(\mathrm{~m}, 2 \mathrm{H}), 2.16(\mathrm{~s}$, 6H), 2.03 (s, 3H), 1.99 (s, 3H), 1.38 (s, 3H), 1.35 (s, 3H); ${ }^{13} \mathrm{C} \mathrm{NMR}\left(\mathrm{CDCl}_{3}, 100 \mathrm{MHz}\right) \delta: 170.3,170.1$ (2C), 169.9, $167.8,158.4,135.3,128.9,128.7,128.3,118.3,110.4$, 99.0, 74.9, 74.5, 73.9, 71.4, 70.5, 68.5, 66.8, 65.3, 60.8, 25.8, 25.6, 20.9, 20.6, 20.5; HRMS (ESI) calcd for $\mathrm{C}_{30} \mathrm{H}_{36} \mathrm{O}_{15} \mathrm{Na}[\mathrm{M}+\mathrm{Na}]^{+}$659.1946, found 659.1936.

\subsubsection{2- $O-\beta-D$-半乳糖基- $L$-抗坏血酸(7)的合成}

化合物 6 (1.91 g, $3 \mathrm{mmol}$ ) 溶于 $50 \mathrm{~mL} 80 \%$ $\mathrm{CH}_{3} \mathrm{COOH}$ 水溶液, $80{ }^{\circ} \mathrm{C}$ 下加热反应 $1 \mathrm{~h}$. 反应停止, 反 应物加入 $50 \mathrm{~mL}$ 乙酸乙酯稀释, 依次用水、饱和 $\mathrm{NaHCO}_{3}$ 溶液洗涤, 中和多余的 $\mathrm{CH}_{3} \mathrm{COOH}$, 有机相用 $\mathrm{MgSO}_{4}$ 干燥, 过滤, 旋干, 柱层析 $[V$ (乙酸乙酯) : $V$ (石 油醚 $)=2: 1]$ 分离得到白色发泡状固体化合物. 向该化 合物(1.61 g, $2.70 \mathrm{mmol}$ )中加入 $0.32 \mathrm{~g}$ 质量分数为 $10 \%$ 的 $\mathrm{Pd} / \mathrm{C}$ 湿剂. 将体系抽成真空状态, 加入甲醇, 通入氢
气，室温下反应 $2.5 \mathrm{~h}$. 反应物抽滤，滤液旋干，柱层析 (乙酸乙酯)得到 $1.30 \mathrm{~g}$ 白色发泡状固体 2-O-(2,3,4,6-四 乙酰基- $\beta$ - $D$-半乳糖基)- $L$-抗坏血酸, 产率 $85 \%$. m.p. $109 \sim 110{ }^{\circ} \mathrm{C} ;[\alpha]_{\mathrm{D}}^{20}-11.03$ (c $\left.1.0, \mathrm{CH}_{3} \mathrm{OH}\right) ;{ }^{1} \mathrm{H}$ NMR $\left(\mathrm{DMSO}-d_{6}, 400 \mathrm{MHz}\right) \delta: 5.28 \sim 5.21(\mathrm{~m}, 3 \mathrm{H}), 5.11 \sim 5.07$ $(\mathrm{m}, 1 \mathrm{H}), 4.80 \sim 4.79(\mathrm{~d}, J=8.8 \mathrm{~Hz}, 1 \mathrm{H}), 4.24(\mathrm{t}, J=6.6$ $\mathrm{Hz}, 1 \mathrm{H}), 4.04$ (ddd, $J=25.4,11.2,6.6 \mathrm{~Hz}, 2 \mathrm{H}), 3.79$ (t, $J=$ $7.0 \mathrm{~Hz}, 1 \mathrm{H}), 3.46 \sim 3.38(\mathrm{~m}, 2 \mathrm{H}), 2.12(\mathrm{~s}, 3 \mathrm{H}), 2.04(\mathrm{~s}$, $3 \mathrm{H}), 1.96$ (s, 3H), 1.92 (s, 3H); ${ }^{13} \mathrm{C}$ NMR (DMSO- $d_{6}, 100$ MHz) $\delta: 170.0,169.9,169.5,169.4,168.6,161.7,117.3$, 99.3, 79.2, 74.8, 70.2, 70.1, 68.7, 68.5, 67.2, 61.8, 60.8, 20.6, 20.5 (2C), 20.4; HRMS (ESI) calcd for $\mathrm{C}_{20} \mathrm{H}_{26} \mathrm{O}_{15} \mathrm{Na}$ $[\mathrm{M}+\mathrm{Na}]^{+}$529.1164, found 529.1170.

$2-O$-(2,3,4,6-四乙酰基- $\beta$ - $D$-半乳糖基)- $L$-抗坏血酸 $(0.50 \mathrm{~g}, 1 \mathrm{mmol})$ 溶于 $30 \mathrm{~mL}$ 甲醇中, 加入 $\mathrm{CH}_{3} \mathrm{ONa}(21.6$ $\mathrm{mg}, 0.4 \mathrm{mmol})$, 室温反应 $3 \mathrm{~h}$. 反应结束加入 $5 \mathrm{~g}$ 的 732 阳离子交换树脂室温搅拌 $30 \mathrm{~min}$. 抽滤, 滤液旋干得到 $0.33 \mathrm{~g}$ 白色发泡状固体 7, 产率 98\%. m.p. 90 91 ${ }^{\circ} \mathrm{C}$; $[\alpha]_{\mathrm{D}}^{20}+36.67\left(c\right.$ 1.0, $\left.\mathrm{H}_{2} \mathrm{O}\right) ;{ }^{1} \mathrm{H} \mathrm{NMR}\left(\mathrm{D}_{2} \mathrm{O}, 600 \mathrm{MHz}\right) \delta$ : $4.64(\mathrm{~d}, J=7.1 \mathrm{~Hz}, 1 \mathrm{H}), 4.53(\mathrm{~d}, J=1.9 \mathrm{~Hz}, 1 \mathrm{H}), 4.01 \sim$ $3.99(\mathrm{~m}, 1 \mathrm{H}), 3.99 \sim 3.87(\mathrm{~m}, 1 \mathrm{H}), 3.78 \sim 3.69(\mathrm{~m}, 4 \mathrm{H})$, $3.67 \sim 3.62(\mathrm{~m}, 3 \mathrm{H}) ;{ }^{13} \mathrm{C}$ NMR $\left(\mathrm{D}_{2} \mathrm{O}, 150 \mathrm{MHz}\right) \delta: 177.7$, $176.5,115.1,103.8,78.3,75.5,72.5,70.7,69.5,68.6,62.4$, 61.0; HRMS (ESI) calcd for $\mathrm{C}_{12} \mathrm{H}_{18} \mathrm{O}_{11} \mathrm{Na}[\mathrm{M}+\mathrm{Na}]^{+}$ 361.0741 , found 361.0748 .

3.2.73- $O-(2,3,4,6$ - 四乙酰基- $\beta-D$ - 葡萄糖基)-5,6- $O$ 异丙叉基- $L$-抗坏血酸 $(8)$ 的合成

将 5,6- $O$-异丙叉基- $L$-抗坏血酸 $(2.00 \mathrm{~g}, 9.26 \mathrm{mmol}$ ) 溶于 $60 \mathrm{~mL} \mathrm{DMF}$ 溶剂中, 随后加入 $\mathrm{K}_{2} \mathrm{CO}_{3}(1.92 \mathrm{~g}, 14$ $\mathrm{mmol}$ )和四乙酰基溴代葡萄糖(5.71 g, $14 \mathrm{mmol})$, 室温下 搅拌反应 $10 \mathrm{~h}$. 停止反应, 反应体系用乙酸乙酯稀释, 水洗分液，水相用乙酸乙酯萃取 1 次，合并有机相，用 柠檬酸溶液洗涤两次, 再用饱和食盐水洗涤一次, 有机 相用无水硫酸镁干燥, 过滤, 滤液旋干, 柱层析分离得 到 $3.31 \mathrm{~g}$ 白色发泡状固体 8, 产率 65\%. m.p. 68 69 ${ }^{\circ} \mathrm{C}$; $[\alpha]_{\mathrm{D}}^{20}+8.70\left(c\right.$ 1.0, $\left.\mathrm{CHCl}_{3}\right) ;{ }^{1} \mathrm{H} \mathrm{NMR}\left(\mathrm{CDCl}_{3}, 400 \mathrm{MHz}\right)$ $\delta: 5.56(\mathrm{~d}, J=8.0 \mathrm{~Hz}, 1 \mathrm{H}), 5.30(\mathrm{t}, J=9.4 \mathrm{~Hz}, 1 \mathrm{H}), 5.20 \sim$ $5.11(\mathrm{~m}, 2 \mathrm{H}), 4.61(\mathrm{~d}, J=2.4 \mathrm{~Hz}, 1 \mathrm{H}), 4.27(\mathrm{dd}, J=12.5$, $5.1 \mathrm{~Hz}, 1 \mathrm{H}), 4.22 \sim 4.17(\mathrm{~m}, 2 \mathrm{H}), 4.12(\mathrm{dd}, J=8.4,6.9 \mathrm{~Hz}$, $1 \mathrm{H}), 4.04$ (dd, $J=8.4,6.6 \mathrm{~Hz}, 1 \mathrm{H}), 3.91$ (ddd, $J=10.1$, 5.0, $2.4 \mathrm{~Hz}, 1 \mathrm{H}), 2.09$ (s, 3H), 2.07 (s, 3H), $2.04(\mathrm{~s}, 3 \mathrm{H})$, $2.03(\mathrm{~s}, 3 \mathrm{H}), 1.35$ (s, 3H), $1.33(\mathrm{~s}, 3 \mathrm{H}) ;{ }^{13} \mathrm{C} \mathrm{NMR}\left(\mathrm{CDCl}_{3}\right.$, $100 \mathrm{MHz}) \delta: 170.7,170.1,169.8,169.5,169.4,144.8$, $120.8,110.4,98.8,74.8,73.2,72.5,72.2,70.7,67.9,65.2$, 61.5, 25.7, 25.6, 20.6, 20.5 (3C); HRMS (ESI) calcd for 
$\mathrm{C}_{23} \mathrm{H}_{30} \mathrm{O}_{15} \mathrm{Na}[\mathrm{M}+\mathrm{Na}]^{+}$569.1477, found 569.1458.

3.2.8 3- $O-\beta-D$-葡萄糖基- $L$-抗坏血酸 (9)的合成

3- $O-\beta$ - $D$-四乙酰葡匋糖基-5,6- $O$-异丙叉基- $L$-抗坏 血酸(1.05 g, $1.92 \mathrm{mmol}$ )溶于 $65 \mathrm{~mL} 80 \% \mathrm{CH}_{3} \mathrm{COOH}$ 溶液 中, $80{ }^{\circ} \mathrm{C}$ 下反应 $2.5 \mathrm{~h}$. 反应停止, 加入 $50 \mathrm{~mL}$ 乙酸乙酯 稀释, 水洗分液, 水相用乙酸乙酯萃取 1 次, 合并有机 相, 依次用水和饱和 $\mathrm{NaHCO}_{3}$ 溶液洗涤至中性, 有机相 用 $\mathrm{MgSO}_{4}$ 干燥, 过滤, 旋干, 柱层析分离得到 $0.79 \mathrm{~g}$ 白 色发泡状产物 3-O- $\beta$ - $D$-四乙酰葡萄糖基- $L$-抗坏血酸, 产率 $80 \%$.

将 3- $O-\beta-D$-四乙酰葡萄糖基- $L$-抗坏血酸 $(0.60 \mathrm{~g}$, $1.16 \mathrm{mmol})$ 溶于 $15 \mathrm{~mL}$ 甲醇中, 随后加入甲醇钠 $(6.28$ $\mathrm{mg}, 0.46 \mathrm{mmol}$ )室温下搅拌反应 $3 \mathrm{~h}$. 停止反应, 反应体 系中加入阳离子交换树脂, 搅拌约 $30 \mathrm{~min}$. 过滤, 滤液 旋干, 得到 $0.38 \mathrm{~g}$ 白色发泡状固体 9, 产率 98\%. m.p. $71 \sim 72{ }^{\circ} \mathrm{C} ;[\alpha]_{\mathrm{D}}^{20}-2.67$ (c 1.0, $\left.\mathrm{H}_{2} \mathrm{O}\right) ;{ }^{1} \mathrm{H}$ NMR $\left(\mathrm{D}_{2} \mathrm{O}\right.$, $600 \mathrm{MHz}) \delta: 5.13(\mathrm{~d}, J=7.9 \mathrm{~Hz}, 1 \mathrm{H}), 4.95(\mathrm{~d}, J=3.1 \mathrm{~Hz}$, $1 \mathrm{H}), 4.08 \sim 4.05(\mathrm{~m}, 1 \mathrm{H}), 3.86(\mathrm{dd}, J=12.5,2.0 \mathrm{~Hz}, 1 \mathrm{H})$, $3.74 \sim 3.67(\mathrm{~m}, 3 \mathrm{H}), 3.54 \sim 3.48(\mathrm{~m}, 2 \mathrm{H}), 3.45 \sim 3.41(\mathrm{~m}$, $2 \mathrm{H}) ;{ }^{13} \mathrm{C}$ NMR $\left(\mathrm{D}_{2} \mathrm{O}, 150 \mathrm{MHz}\right) \delta: 177.2,171.1,142.5$, 133.1, 101.5, 76.3, 76.0, 75.4, 73.1, 69.2, 69.0, 62.4, 60.4; HRMS (ESI) calcd for $\mathrm{C}_{12} \mathrm{H}_{18} \mathrm{O}_{11} \mathrm{Na}[\mathrm{M}+\mathrm{Na}]^{+} 361.0748$, found 361.0741 .

\section{3 生物活性测试}

\section{$3.3 .1 \alpha$ - 葡萄糖苷酶活性测试}

采用 96 孔板法 ${ }^{[12]}$, 每孔取 $50 \mu \mathrm{L}$ 的待测溶液 (AA-2 $\beta \mathrm{G} 、 \mathrm{AA}-2 \beta \mathrm{Gal}$ 和阿卡波糖浓度为 $0.75,1.5,3,7.5$, $15,30 \mathrm{mmol} / \mathrm{L} ; \mathrm{AA}-3 \beta \mathrm{G}$ 浓度为 $30,44,59,74,118,148$ $\mathrm{mmol} / \mathrm{L}$ ), 加入 $50 \mu \mathrm{L}$ 磷酸二氢钠缓冲溶液( $\mathrm{PBS}, \mathrm{pH} 6.5)$ 及 $10 \mu \mathrm{L}$ 的酿酒酵母源 $\alpha$-葡萄糖苷酶液 $(0.45 \mathrm{U} / \mathrm{mL})$, $37{ }^{\circ} \mathrm{C}$ 恒温预热 $15 \mathrm{~min}$, 加入 $50 \mu \mathrm{L}$ 的 $p \mathrm{NPG}$ 溶液 $(0.83$ $\mathrm{mmol} / \mathrm{L})$, 在 $37{ }^{\circ} \mathrm{C}$ 恒温条件下继续反应 $15 \mathrm{~min}$. 加入 $100 \mu \mathrm{L} 1 \mathrm{~mol} / \mathrm{L}$ 的碳酸钠溶液终止反应. 然后用酶标仪 测定 $405 \mathrm{~nm}$ 下吸光度 $D$ 值. 实验以 PBS 代替待测样品 为空白对照组, 阿卡波糖作为阳性对照组. 阴性对照组: PBS 代替对硝基苯基 $\alpha-D$-葡萄糖苷 $(p N P G)$ 对照组, 在 $405 \mathrm{~nm}$ 处无明显吸收.

样品对 $\alpha$-葡萄糖苷酶抑制率的计算公式为: 抑制率 $(\%)=\left(D_{\text {空白 }}-D_{\text {样品 }}\right) / D$ 空白 $\times 100 \%, D_{\text {样品表示样品的吸光度 }}$ 值(排除样品自身的干扰), D 空白表示空白的吸光度值, 每 组实验重复三组平行, 抑制率取平均值. 以浓度为横坐 标, 抑制率为纵坐标作图得到各样品的 $\mathrm{IC}_{50}$ 值.

\section{$3.3 .2 \alpha$ - 葡萄糖苷酶抑制类型测定}

参考文献方法 ${ }^{[13]}$, 固定酿酒酵母源 $\alpha$-葡萄糖苷酶 的浓度为 $0.45 \mathrm{U} / \mathrm{mg}$, 配置 AA- $2 \beta \mathrm{G}$ 浓度分别为 $0.75 、 3 、$
$7.5 \mathrm{mmol} / \mathrm{L}$; AA-2 $\beta$ Gal 浓度分别为 $1.5 、 3 、 7.5 \mathrm{mmol} / \mathrm{L}$; $\mathrm{AA}-3 \beta \mathrm{G}$ 浓度分别为 74、118、148 mmol/L, 分别在这些 浓度下, 改变底物 $p \mathrm{NPG}$ 浓度分别为 $0.3 、 0.43 、 0.6 、 1.2$ $\mathrm{mmol} / \mathrm{L}$. 反应每隔 $1 \mathrm{~min}$ 记录吸光度值随时间推移而增 长的曲线. 计算反应的初速度及其倒数与底物的 $p \mathrm{NPG}$ 浓度倒数进行作图, 得到 Lineweaver-Burk 曲线.

\subsection{3 $\alpha$-淀粉酶活性测试}

参考文献方法 ${ }^{[14]}$, 取 $0.25 \mathrm{~mL}$ 猪胰腺 $\alpha$-淀粉酶溶液 (3.54 U/mL), 加入 $0.25 \mathrm{~mL}$ 待测样品溶液 $(0.015 、 0.077$ 、 $0.15 、 0.77 \mathrm{mmol} / \mathrm{L})$, 在 $37{ }^{\circ} \mathrm{C}$ 的温度下预热 $10 \mathrm{~min}$, 然 后加入 $0.5 \mathrm{~mL}$ 的淀粉溶液在 $37{ }^{\circ} \mathrm{C}$ 水浴加热 $3 \mathrm{~min}$. 完 毕后加入 $0.5 \mathrm{~mL} \mathrm{DNS}$ 显色溶液, 混合均匀, 置于 $80{ }^{\circ} \mathrm{C}$ 沸水浴中反应 $8 \mathrm{~min}$. 反应完毕将体系冷却至室温, 加 入 $5 \mathrm{~mL}$ 蒸馏水进行稀释. 显色溶液在 $540 \mathrm{~nm}$ 波长下进 行吸光值检测. 空白对照: 用 PBS $(\mathrm{pH}=6.9)$ 代替待测 样品进行上述反应. 样品对猪胰腺 $\alpha$-淀粉酶抑制率的计 算公式为: 抑制率 $(\%)=(D$ 空白 $-D$ 样品 $) / D$ 空白 $\times 100 \%, D$ 样品 表示样品的吸光度值(样品无背景吸收), $D$ 空白表示空白 的吸光度值, 每组实验测三个平行, 抑制率取平均值.

辅助材料(Supporting Information) 化合物 3 9 的核 磁共振氢谱和碳谱谱图. 这些材料可以免费从本刊网站 (http://sioc-journal.cn/)上下载.

\section{Referenes}

[1] (a) Zhu, J.; Liu, W.; Yu, J.; Zou, S.; Wang, J.; Yao, W.; Gao, X. Carbohydr. Polym. 2013, 98, 8.

(b) Zhou, Z.; Yan, J.; Tang, X.; Zhang, W.; Zhang, Y.; Chen, X.; Su, X.; Yang, D. Chin. J. Org. Chem. 2010, 30, 582 (in Chinese). (周祖文, 晏菊芳, 唐雪梅, 张蔚瑜, 张映霞, 陈欣, 苏小燕, 杨 大成, 有机化学, 2010, 30, 582.)

[2] Ruan, C.-T.; Lam, S.-H.; Lee, S.-S.; Su, M.-J. Phytomedicine 2013, $20,667$.

[3] (a) Aoki, K.; Muraoka, T.; Ito, Y.; Togashi, Y.; Terauchi, Y. Intern. Med. 2010, 49, 1085.

(b) Derosa, G.; Maffioli, P. Clin. Ther. 2012, 34, 1221.

(c) Hollander, P. Drugs 2012, 44, 47.

[4] (a) Escandon-Rivera, S.; Gonzalez-Andrade, M.; Bye, R.; Linares, E.; Navarrete, A.; Mata, R. J. Nat. Prod. 2012, 75, 968.

(b) Huang, Y.; Fu, W.; Li, Y.; Liu, Yu.; Li, Z.; Liu, Y.; Liu, S.; Sun, J.; Li, N.; Wang, B.; Gao, X.; Zhang, D. J. Evidence-Based Complementary Altern. 2014, 2014, 9.

(c) Kim, Y.-M.; Wang, M.-H.; Rhee, H.-I. Carbohydr. Res. 2004, 339, 715 .

(d) Alarcon-Aguilara, F. J.; Roman-Ramos, R.; Perez-Gutierrez, S.; Aguilar-Contreras, A.; Contreras-Weber, C. C.; Flores-Saenz, J. L. J. Ethnopharmacol. 1998, 61, 101.

[5] (a) Cheng, J.; Zhou, Z.-W.; Sheng, H.-P.; He, L.-J.; Fan, X.-W.; He, Z.-X.; Sun, T.; Zhang, X.; Zhao, R. J.; Gu, L.; Cao, C.; Zhou, S.-F. Drug Des., Dev. Ther. 2015, 9, 33.

(b) Cai, H.; Liu, F.; Zuo, P.; Huang, G.; Song, Z.; Wang, T.; Lu, H.; Guo, F.; Han, C.; Sun, G. Med. Chem. 2015, 11, 383.

[6] Toyoda-Ono, Y.; Maeda, M.; Nakao, M.; Yoshimura, M.; Sugiura-Tomimori, N.; Fukami, H. J. Agric. Food Chem. 2004, 52, 2092.

[7] (a) Takebayashi, J.; Yagi, Y.; Ishii, R.; Abe, S.; Yamada, K.; Tai, A. Biosci. Biotechnol. Biochem. 2008, 72, 1558. 
(b) Zhang, Z.; Liu, X.; Zhang, X.; Liu, J.; Hao, Y.; Yang, X.; Wang, Y. Arch. Pharm. Res. 2011, 34, 801.

(c) Zhang, Z.; Zhang, L.; Hao, Y. J. Practic. Med. 2011, 27, 11 (in Chinese).

(张自萍，张立平，郝艳芳，实用医学杂志，2011，27，11.)

(d) Li, H.; Zhang, Z.; Liao, G. Chin. J. New Drug 2007, 16, 212 (in Chinese).

(李弘武, 张自萍，廖国玲，中国新药杂志, 2007, 16, 212.)

(e) Xie, R.; Zhang, Z.; Qiu, J. Bull. Sci. Technol. 2010, 26, 362 (in Chinese).

(谢若男，张正波，震娟萍，科技通报，2010, 26, 362.)

[8] Zhang, Z.; Liu, X.; Wu, T.; Liu, J.; Zhang, X.; Yang, X.; Goodheart, M. J.; Engelhardt, J. F.; Wang, Y. Cell Biol. Toxicol. 2011, 27, 107.

[9] (a) Olabisi, A. O.; Wimalasena, K. J. Org. Chem. 2004, 69, 7026. (b) Zheng, H. Pharmaceutical Chemistry, People's Medical Publishing House, Bejing, 2007, p. 426 (in Chinese). (郑虎，药物化学，人民卫生出版社，北京，2007，p. 426.)

[10] Cho, B.-H.; Kim, J.-H.; Jeon, H.-B.; Kim, K.-W. Tetrahedron 2005, $61,4341$.

[11] Kim, G.-N.; Kwon, Y.-I.; Jang, H.-D. J. Med. Food 2011, 14, 712.

[12] (a) Balan, K.; Perumal, P.; Sundar abaalaji, N.; Palvannan, T. $J$. Mol. Struct. 2015, 1081, 62.

(b) Sheng, Z.; Dai, H.; Pan, S.; Wang, H.; Hu, Y.; Ma, W. Molecules 2014, 19, 10563.

[13] Brindis, F.; Rodríguez, R.; Bye, R.; Gonzalez-Andrade, M.; Mata, R. J. Nat. Prod. 2011, 74, 314.

[14] Thongprajukaew, K.; Choodum, A.; Sa-E, B.; Hayee, U. Food Chem. 2014, 163, 87.

(Li, L.; Fa, Y.) 